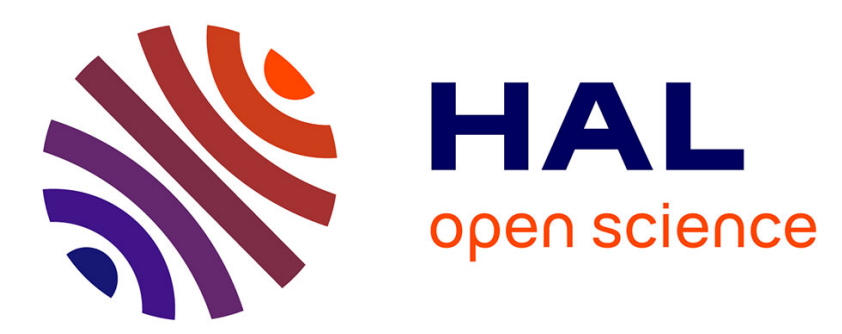

\title{
Core cognition in adult vision: A surprising discrepancy between the principles of object continuity and solidity.
} Andreas Falk, Ghislaine Labouret, Véronique Izard, Annie E Wertz, Frank C. Keil, Brent Strickland

\section{- To cite this version:}

Andreas Falk, Ghislaine Labouret, Véronique Izard, Annie E Wertz, Frank C. Keil, et al.. Core cognition in adult vision: A surprising discrepancy between the principles of object continuity and solidity.. Journal of Experimental Psychology: General, 2020, 10.1037/xge0000785 . hal-02997465

\section{HAL Id: hal-02997465 \\ https://hal.science/hal-02997465}

Submitted on 10 Nov 2020

HAL is a multi-disciplinary open access archive for the deposit and dissemination of scientific research documents, whether they are published or not. The documents may come from teaching and research institutions in France or abroad, or from public or private research centers.
L'archive ouverte pluridisciplinaire HAL, est destinée au dépôt et à la diffusion de documents scientifiques de niveau recherche, publiés ou non, émanant des établissements d'enseignement et de recherche français ou étrangers, des laboratoires publics ou privés. 
Article accepted for publication in Journal of Experimental Psychology: General (03/25/2020).

Please do not cite this manuscript, see full published version at

http://dx.doi.org/10.1037/xge0000785

Core cognition in adult vision: A surprising discrepancy between the principles of object continuity and solidity.

Andreas Falck* ${ }^{1,2}$, Ghislaine Labouret* ${ }^{3,4}$, Véronique Izard ${ }^{4}$, Annie E. Wertz ${ }^{5}$, Frank C. Keil ${ }^{6}$, and Brent Strickland ${ }^{1,7}$

${ }^{1}$ Institut Jean Nicod, Département d'études cognitives, ENS, EHESS, CNRS, PSL University, Paris, France; ${ }^{2}$ Department of psychology, Lund University, Lund, Sweden; ${ }^{3}$ Laboratoire de Sciences Cognitives et Psycholinguistique, Département d'Etudes Cognitives, ENS, EHESS, CNRS, PSL University, Paris, France; ${ }^{4}$ Integrative Neuroscience and Cognition Center, CNRS, Université de Paris; ${ }^{5}$ Max Planck Institute for Human Development, Max Planck Research Group Naturalistic Social Cognition, Berlin, Germany; ${ }^{6}$ Department of Psychology, Yale University; ${ }^{7}$ School of Collective Intelligence, UM6P, Ben Guerir, Morocco.

*These authors contributed equally.

CAmerican Psychological Association, 2020. This paper is not the copy of record and may not exactly replicate the authoritative document published in the APA journal. Please do not copy or cite without author's permission. The final article is available, upon publication, at: http://dx.doi.org/10.1037/xge0000785 


\begin{abstract}
From an early age, humans intuitively expect physical objects to obey core principles, including continuity (objects follow spatiotemporally continuous paths) and solidity (two solid objects cannot occupy the same space at the same time). These two principles are sometimes viewed as deriving from a single overarching "persistence" principle. Indeed, violations of solidity where one solid object seemingly passes through another could theoretically be interpreted as a violation of continuity, with an object "teleporting" to switch places rather than passing through a solid obstacle. However, it is an empirical issue whether the two principles are processed distinctly or identically to one another. Here, adult participants tracked objects during dynamic events in a novel location detection task, which sometimes involved violations of the principles of continuity or solidity. While participants explicitly noticed both types of violations and reported being equally surprised at both, they made more errors and answered more slowly after continuity violations than after solidity violations. Our results demonstrate that the two principles show different signature patterns, and are thus represented distinctly in the mind.
\end{abstract}

Keywords: object tracking, core knowledge, naïve physics, perception, cognitive development. 
Humans expect physical objects to behave according to a set of basic physical laws, beginning in early infancy (see Baillargeon, 2004 for a review). For example, infants expect that objects will obey the principles of cohesion (i.e., objects maintain a single bounded contour over time; e.g. Spelke, 1990), continuity (i.e., objects cannot disappear into thin air or teleport from one location to another; e.g. Spelke, Breinlinger, Macomber, \& Jacobson, 1992), and solidity (i.e., two solid objects cannot occupy the same space at the same time; e.g. Spelke et al., 1992). The issue at stake in the current paper is how such physical constraints are represented during adults' real time processing of dynamic events. Is there single underlying principle (such as a "persistence principle"; Baillargeon, 2008) responsible for generating expectations on the fly or are there representations of multiple underlying principles (e.g. continuity vs. solidity)? Here, inspired by recent work showing that "core physics" from infancy continues to influence adult response patterns in "high pressure" perceptual tasks (e.g. Strickland \& Scholl, 2015), we devised a novel experimental paradigm which can directly assess this question.

The evidence that pre-verbal infants possess an ability to represent basic characteristics about the behavior of physical objects is no longer controversial. For example, Baillargeon and DeVos (1991) presented 3.5-month-old infants with an object moving behind a screen, which had a window cut. Infants expected the object to be visible in this window before it could reappear on the other side, demonstrating an understanding of continuity (i.e. that teleportation is not possible). Likewise, other experiments provide evidence that preverbal infants display a precocious understanding of the solidity principle (Baillargeon, 1986; 1987). For example, Baillargeon (1986) showed 6-8 months old infants a toy car passing behind an occluder and reappearing on its other side. Infants looked longer at events where a box was placed on the car's tracks but the car reappeared on the other side, compared to events with no box or with the box 
placed behind the tracks. This suggests that they expected the car to be stopped by the box (consistent with the solidity principle) and not to magically teleport from one location to another (consistent with the continuity principle).

Subsequent research has demonstrated that such "core knowledge" does not simply disappear when we grow up, but instead that core principles from infancy serve to guide and constrain perceptual processing in adults (Cheries, Mitroff, Wynn, \& Scholl, 2009; Shurgin \& Flombaum, 2017; Strickland \& Scholl, 2015). Just as infant object and quantity tracking is impaired by violations of object cohesion (Cacchione \& Amici, 2015; Cheries, Mitroff, Wynn, \& Scholl, 2008; Huntley-Fenner, Carey \& Solimando, 2002), adults are similarly impaired in tracking multiple moving objects that violate this principle (vanMarle \& Scholl, 2003; Mitroff, Scholl, \& Wynn, 2004). Again analogously to infant results showing that infants are highly sensitive to violations of object continuity (Baillargeon \& DeVos, 1991), violations of this principle also impair object tracking through occlusion for adults performing a color change detection task (Flombaum \& Scholl, 2006).

The current study exploits these traces of core physical cognition in adulthood in order to shed light on a foundational theoretical issue regarding the nature of core knowledge and its place in cognitive architecture. The issue is whether the principles of cohesion, continuity, and solidity are actually represented via a single overarching "persistence" principle (Baillargeon, 2008) or whether some or all of these principles are represented piecemeal, having unique functional roles. As a way of addressing this broader theoretical question, we concentrate here on examining the possible unity, or possible distinction of the continuity and solidity principles in on-line processing. 
In discussions surrounding the principles of core physics, researchers in the field of infant cognition have emphasized the similarity between continuity and solidity, mostly due to considerations of theoretical parsimony (e.g. Spelke, 1994). Indeed, any event presenting a violation of the principle of solidity can also be interpreted as a violation of spatio-temporal continuity: for example, a scene showing a solid object seemingly passing through a barrier could also be viewed as an object disappearing and then coming back to existence on the opposite side of the barrier (Figure 1). Consistent with this perspective, infants can detect physical violations of both continuity and solidity as early as 3.5 months (Baillargeon, 1987; Carey, 2011), suggesting a parallel in the time course of development and thus a possible common underlying mechanism. 

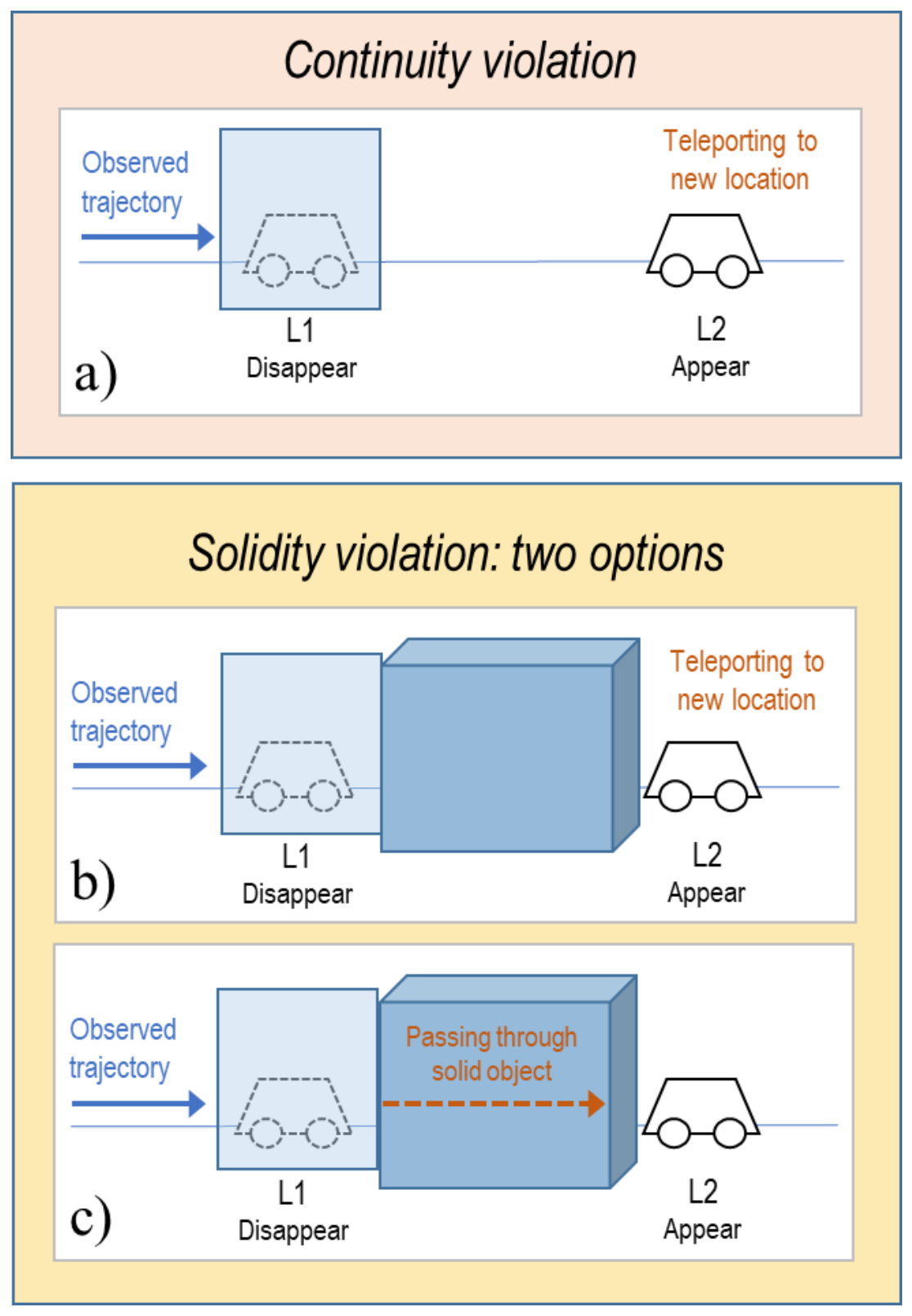

Figure 1. Possible mechanisms for processing continuity vs. solidity violations, exemplified by scenes in which the depicted car is seen travelling behind an opaque occluder at (L1), and re-appears "magically" at another location (L2). For continuity (a), an expectation that objects persist in time and space (i.e. that they would not teleport) is sufficient to view this as a violation. Solidity violations can either be processed the same way (b), or may involve an alternative constraint that "solid objects do not pass through each other" (c).

A variety of studies and theoretical work however suggest that the two principles of solidity and continuity may be processed separately. In contrast with the idea of a single 
"persistence" principle, Scholl and Leslie (1999) proposed that core object knowledge consists of two distinct representational systems: spatiotemporal knowledge (how objects move in time and space, encompassing the continuity principle) and contact-mechanical knowledge (how mechanical interactions between objects work, including solidity constraints). In line with this distinction, toddlers show striking discrepancies in performance between search paradigms: they succeed on purely spatiotemporal tasks, such as retrieving hidden crackers from the box that had received the most crackers earlier (Feigenson, Carey, \& Hauser, 2002), but fail on tasks that require reasoning about mechanical interactions between objects (Hood, Carey, \& Prasada, 2000; Keen, 2003). Further supporting this hypothesis, Santos (2004) also showed that non-human primates succeed on a spatiotemporal search task, but fail on an identical task that incorporates contact-mechanics.

Research on adult visual illusions also suggests a discrepancy in how the principles of solidity and continuity are processed. For example, in the Pulfrich double pendulum illusion (Leslie, 1988; Wilson \& Robinson, 1986; see Figure 2), adults readily tolerate an apparent violation of object solidity. In this illusion, two pendulums swing in opposite directions in the frontal plane but seem to follow elliptic courses when viewed with both eyes, one of which is covered by a low density light filter (which influences stereoscopic cues to depth). The pendulums' arms then appear to pass through one another. In cases like this, the brain has a choice between applying the solidity principle to infer the correct object trajectories, or allowing violations of solidity while preserving the apparent elliptical motion. It appears to use the latter strategy, thus suggesting that in at least some perceptual contexts, object solidity (in contrast to object continuity) is not given a high priority. 

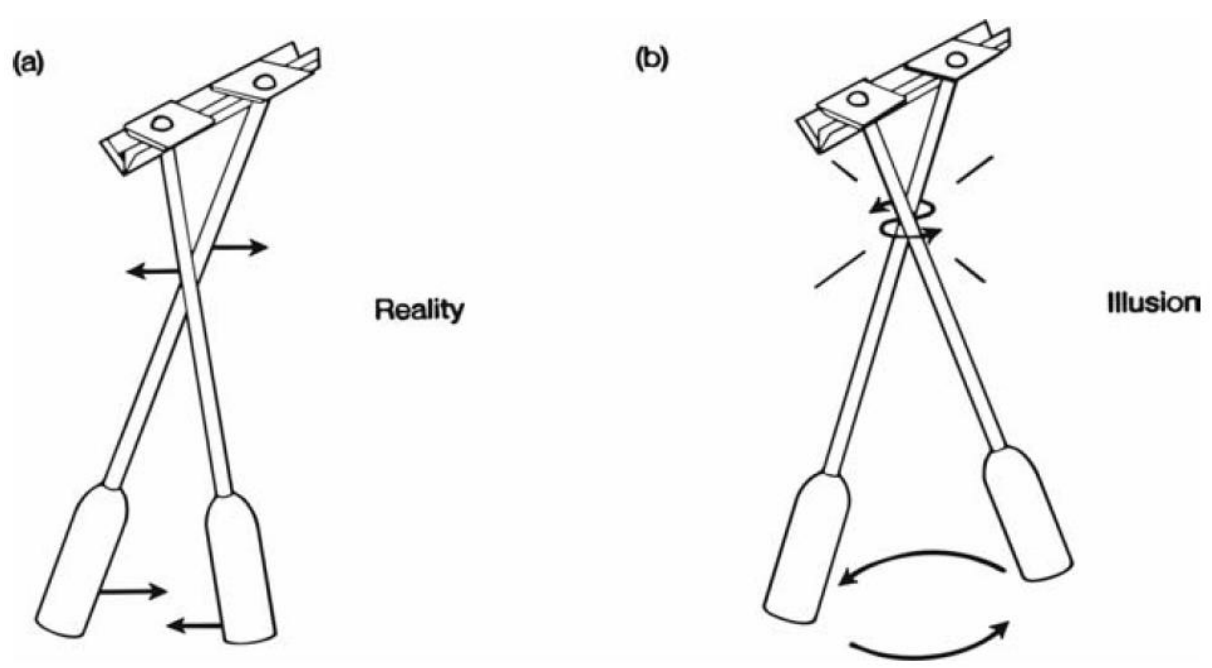

Figure 2. The Pulfrich double pendulum illusion: (a) what really happens, and (b) how the illusion appears to an observer viewing with reduced luminance to one eye (direction of apparent rotation depends upon which eye). Reproduced from Leslie (1988).

While these results are interesting and suggestive, the range and type of experimental paradigms employed make direct comparison between the continuity and solidity principles difficult. Therefore, here we developed a new task to compare the influence of these principles directly, using minimally contrasting experimental conditions. Adult participants were presented with events where one object disappeared in one location and later reappeared in another (Figure 1). If, as previous researchers have suggested, the principles of continuity and solidity are reducible to each other, then processing this apparent "teleportation" on-line would be equally difficult regardless of whether there was a solid obstacle in the way of the object trajectory, or whether the object moved invisibly from one location to another without such a solid obstacle in its path. However if continuity and solidity are processed in categorically different ways, we might also expect important differences in response patterns when people are confronted with violations of solidity vs. continuity. 


\section{Experiment 1}

In Experiment 1, adult participants tracked an object in different events presenting either violations or non-violations of the continuity or solidity principles. We were interested in assessing whether participants would err more and/or show slower response times in response to violations of either principle. We also wished to investigate to what extent these core principles would be affected by learning contexts: would continuity or solidity violations be easier to integrate over time ? In order to examine possible differential effects on learning, we varied the ratio of violations to non-violations as an additional factor.

\section{Method}

This study was part of a project approved by the ethical research committee from Paris Descartes University (CERES), and all participants provided informed consent.

\section{Participants}

120 U.S. based participants were recruited online via Amazon's Mechanical Turk. Participants were compensated a small amount. Sample size was determined via a power analysis based on the effect size from a pilot study (ANOVA repeated measures, within-between interaction, two groups with two measures each, $\mathrm{f}=.25, \alpha=.05$, power $=95 \%$ ), using the G*Power 3 software (Faul, Erdfelder, Lang, \& Buchner, 2007): estimated sample size was 106, which we rounded up to 120 to account for a predicted $10 \%$ exclusion rate. Indeed, 13 participants $(11 \%)$ were excluded from analysis because they did not follow instructions or encountered technical issues, as per pre-defined exclusion criteria available in the supplemental material (Table S1). Furthermore, an additional 67 trials (1.3\%) were excluded from analysis because of an issue with the video display, or because the participant was not paying attention 
(Table S3). For the 107 participants included, ages ranged from 20 to 73 years $($ mean $=36.2), 44$ (41\%) were women and 90 (84\%) were right-handed.

Stimuli

We created photorealistic 3D-animations depicting a toy car on a flat surface, with a wall in the background. Two familiarization videos showed the car starting from a side of the screen, then moving horizontally in a straight line. It then either slowed down to a halt near the other side of the screen (continuity familiarization), or bumped into a solid block placed on its path (solidity familiarization). Both familiarization videos were shown once to introduce participants to the displays.

In test videos (see Figure 3), the trajectory of the car was partially occluded by two occluders. At the beginning of a trial, the occluders were down, so participants could see that there was nothing behind them. A partially visible static car was positioned on the far side of the screen. The occluders then raised and the car started moving on a horizontal trajectory. Shortly after the car had stopped behind an occluder, both occluders dropped simultaneously, revealing the car either in the physically possible or in the physically impossible position.

In continuity events (see Figure 3a), the car passed behind the first occluder, then was seen traveling in the gap between the two occluders, before stopping behind the second one. When the occluders dropped, the car was revealed either behind the second occluder (nonviolation condition), or behind the first one (a violation of the continuity principle). A flat blue surface was added to the back wall to match the color contents of the solidity videos. 
a. Continuity
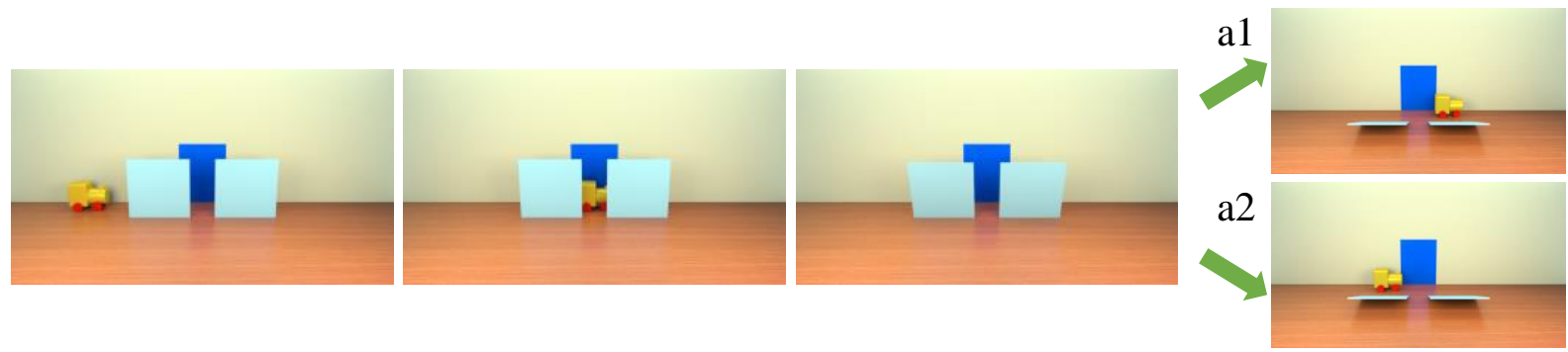

b. Solidity
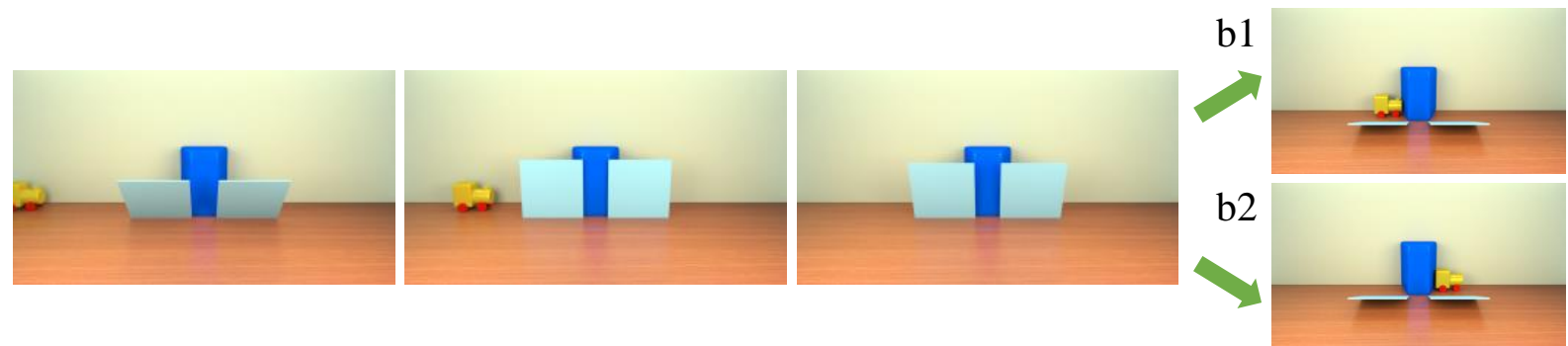

Figure 3. Frames from the continuity (a) and solidity (b) videos: first frame (left), car motion (center) and two reveal possibilities (top-right, 1: non-violation; bottom-right, 2: violation). Full videos are available online at https://goo.gl/Ldjiej.

In solidity events (see Figure 3b), the setting was identical, except that there was a solid block between the two occluders. The back of the block came in contact with the wall, so that it was clear that the car could not pass behind it. As the occluders dropped, the car was revealed either just before the obstacle (non-violation), or just behind it, as if it had traversed the block (a violation of the solidity principle).

This resulted in four video types: two principles (continuity, solidity) crossed with two violation statuses (violation, non-violation). Furthermore, mirror videos were created to counterbalance direction of movement (from left to right and reverse). On half of the trials for each condition, the car moved from left to right, and on the other half it moved from right to left. 
Thus, the left and the right positions were not systematically associated with violations or nonviolations, in both the continuity and the solidity conditions.

Detailed video timelines are presented in the supplemental material (Figure S1). Speed of movement, duration of object disappearance (1s), and object reveal time (as measured from the beginning of the video) were equated across conditions. To that avail, the car started moving slightly later $(667 \mathrm{~ms})$ in solidity events than in continuity events.

Videos were $854 \times 480$ pixels wide, centered in an otherwise empty browser window, except for a reminder of the answer keys and the trial number. Actual display size was not controlled and depended on the participant's computer screen size.

\section{Procedure}

Participants used their own computer to perform the experiment, which was made available online through the Qualtrics online survey platform (Qualtrics, Provo, UT). On each trial, participants were asked to indicate the position of the car after the occluders had dropped (left or right side, 2-alternatives forced choice). They answered by pressing $\mathrm{J}$ for left and $\mathrm{K}$ for right, with the index and middle fingers of their dominant hand. Response time was measured from the first video frame where the car was visible after the occluders started dropping.

In our task, participants needed simply to respond based on the position of the car as soon as they detected it after removal of the occluders. We nonetheless hypothesized that participants would process the trajectory of the car automatically, and that they would err more when the position of the car did not match their expectations. Two aspects of the task were designed to increase our chances of detecting such anticipation errors. First, instructions emphasized speed over accuracy. Warnings were displayed for response times longer than 500ms and when the participant tried to answer too early. Second, we made the trajectory of the car predictive of its 
final position by manipulating the frequency of violations and non-violations trials. Participants were randomly assigned to one of two violation context conditions, where violations were either non-frequent (25\% of trials) or frequent (75\%). Manipulating whether violations or nonviolations were most frequent allowed us to investigate to what extent participants would learn to adapt to violations over time.

After reading the instructions and watching the familiarization videos, participants performed four training trials, with the four non-violation videos (condition $\times$ direction of motion). If they made two or more mistakes, a reminder of the instructions was displayed. Following training, they performed 3 blocks of 16 test trials each. Within each block, the factors of direction of movement (from left to right or reversed), principle (continuity or solidity, depending on the absence or presence of an obstacle on the object's path), and violation status (depending on whether the object's final position was compatible with the laws of physics or not) were fully crossed.

Depending on the violation context, each block comprised 12 non-violations and 4 violations, or 12 violations and 4 non-violations. To establish the context, the four first test trials corresponded to frequent trials (e.g. four non-violations in the $25 \%$ violations condition) and showed all four possible variants of the frequent trials ( 2 principles $\times 2$ directions). They were followed by a rare trial type in the fifth position (e.g. a violation in the $25 \%$ violations condition). This rare trial was selected from one of these four possible trial types, counterbalanced across participants.

After completing the experiment, participants were asked if they noticed the physical violations and which type they found more surprising. First, we asked "Did you ever notice that the car did something physically impossible? If so, what did it do?". Then, on a separate page to 
prevent modification of the answer to the previous question, we asked: "Did you ever get the impression that the car went through the solid block?", "Did you ever get the impression that the car appeared in a location that it should not have appeared in, by 'popping out and into existence'?", and "Was one type of physical violation more surprising or inexplicable than the other? Which one?". We also asked participants additional questions that were not analyzed with respect to our hypotheses but used for making sure the experiment went fine: did they encountered any issue, did they use any special strategy, and how well they thought they performed (in term of giving the correct left/right answer).

\section{Results}

Data and analysis scripts are available online at https://osf.io/9daxp/.

\section{Detection accuracy}

Even though they were asked to respond with the car's location once they could see it on the screen, participants sometimes answered incorrectly. More errors were made on violation events, corresponding to the participants answering according to their expectation of the car's location based on physical principles. Indeed, the overall accuracy was $89.9 \% \pm 2.6 \%$ (95\% CI) for violations and $97.2 \% \pm 1.2 \%$ for non-violations, a significant difference, $F(1,106)=25.5$, $p<.001, \eta_{\mathrm{p}}^{2}=.194,95 \%$ CI $[.075, .319]$. Furthermore, when they answered correctly, participants were $16 \mathrm{~ms}$ slower on violations ( $384 \mathrm{~ms} \pm 10 \mathrm{~ms}$, mean of individual medians) than non-violations $(368 \mathrm{~ms} \pm 10 \mathrm{~ms}), F(1,105)=16.8, p<.001, \eta^{2} \mathrm{p}=.138,95 \%$ CI $[.038, .259]$. 


\section{Experiment 1 - Accuracy}

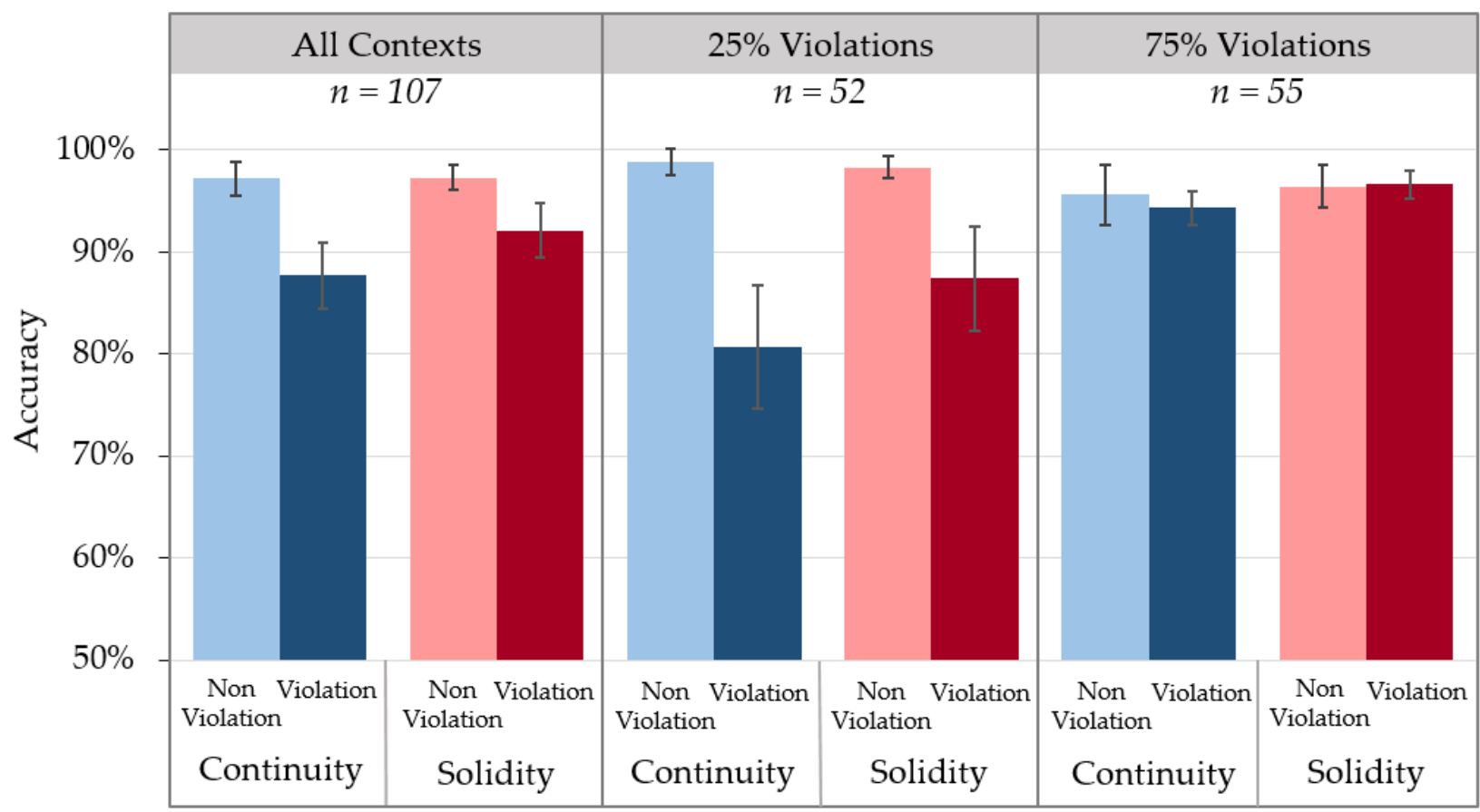

Figure 4. Experiment 1 mean accuracies, depending on Violation Context (facet), Principle and

Violation (abscissa and color). Error-bars represent 95\% confidence intervals.

Importantly, the effect of violation differed across the two principles, as indicated by a two-way Principle $\times$ Violation interaction $\left(F(1,105)=7.31, p=.008, \eta^{2} \mathrm{p}=.065,95 \%\right.$ CI $[.005$, .171], three-way ANOVA with factors of Context, Principle and Violation). This interaction was created by a larger drop in accuracy for violations in continuity (violations $87.7 \%$ vs. nonviolations $97.2 \%$ ) than in solidity $(92.1 \%$ vs. $97.3 \%)$, indicating stronger anticipations that the objects would obey the principle of continuity than solidity. The three-way interaction between Context, Principle and Violation was not significant, $F(1,105)=3.01, p=.086, \eta_{\mathrm{p}}^{2}=.028,95 \%$ CI $[.0, .114]$. However, the observed two-way interaction between Principle and Violation was present only in the $25 \%$ violation context, $F(1,51)=6.42, p=.014, \eta^{2} \mathrm{p}=.112,95 \%$ CI $[.004$, $.280]$, but not in the $75 \%$ violation context, $F(1,54)=0.902, p>.250, \eta^{2} \mathrm{p}=.016,95 \% \mathrm{CI}[.0$, 
.132]. In general, the effect of violations largely vanished in the $75 \%$ violations context, with participants responding at ceiling in all conditions (two-way interaction between Violation and Context: $\left.F(1,105)=29.6, p<.001, \eta^{2} \mathrm{p}=.220,95 \% \mathrm{CI}[.094, .346]\right)$, masking any potential difference between principles in this context.

\section{Experiment 1 - Response Time for Correct Answers}

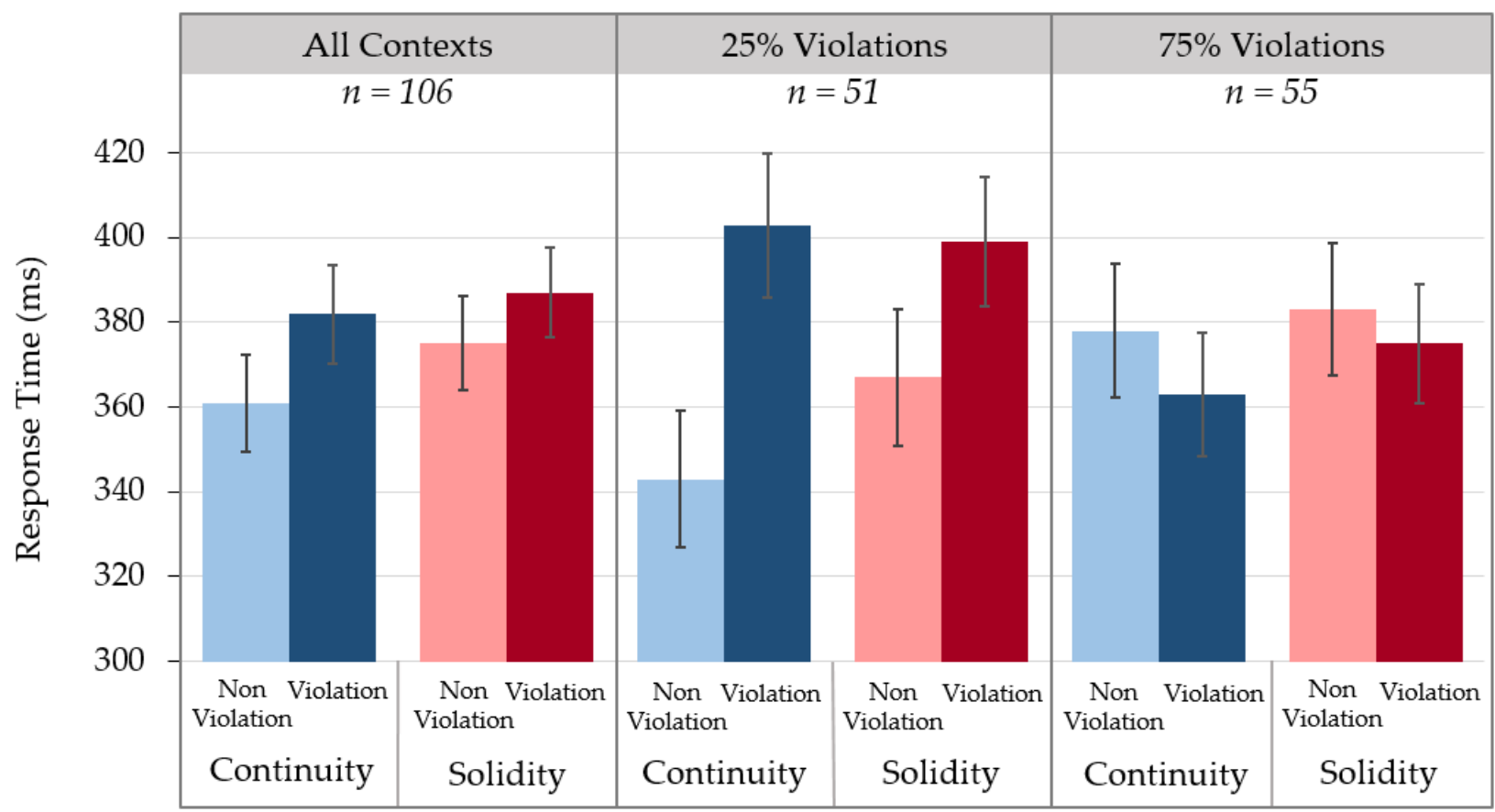

Figure 5. Experiment 1 response times for correct answers (means of individual medians), depending on Learning Context (facet), Principle and Violation (abscissa and color). Error-bars represent $95 \%$ confidence intervals.

\section{Response times}

Similar trends were observed in the analysis of Response times for correct responses (see Figure 5; one outlier was excluded from RT analysis, as per criteria in table S1). For response times the 2-way interaction between Principle and Violation was not significant $(F(, 104)=2.26$, $\left.p=.136, \eta^{2} \mathrm{p}=.021,95 \% \mathrm{CI}[0, .104]\right)$, however, we found a 3-way interaction between Context, 
Principle and Violation $\left(F(1,104)=6.87, p=.010, \eta_{\mathrm{p}}^{2}=.062,95 \%\right.$ CI $\left.[.004, .167]\right)$. Planned comparisons revealed an effect agreeing with that for accuracy in the $25 \%$ violations context, in which RTs increased more for continuity violations compared to non-violations $(+59 \mathrm{~ms}, 95 \% \mathrm{CI}$ $[40,78])$, than for solidity violations compared to solidity non-violations (+32ms, 95\% CI [22, 42]). In other words, just as people showed a greater drop in accuracy in continuity violations (relative to non-violations) than for solidity, they also showed a greater increase in response times for continuity violations (relative to non-violations) than for solidity. This produced a significant two-way Principle $\times$ Violation interaction within the $25 \%$ violations context, $F(1,50)=8.69, p=.005, \eta^{2} \mathrm{p}=.148,95 \% \mathrm{CI}[.015, .323]$. In the $75 \%$ violations context, RTs were comparable across principles, with correct responses to violations (frequent case) becoming slightly faster than correct responses to non-violations $(15 \pm 14 \mathrm{~ms}$ for continuity, $7 \pm 12 \mathrm{~ms}$ for solidity), failing to produce a two-way Principle $\times$ Violation interaction in this context, $F(1,54)=0.618, p>.250, \eta_{\mathrm{p}}^{2}=.011,95 \%$ CI $[0, .119]$. Both Accuracy and Response Times show that violations affect performance differently for the continuity and solidity events, and these results are driven by the $25 \%$ violation context.

\section{Dynamics over time}

To look more into the dynamics of the effect, we examined the evolution of accuracy and response times across trials. In all conditions, accuracy was low on the very first violation trial, then quickly went up (mean accuracy $57.7 \% \pm 9.7 \%$ on the first violation, versus $80.2 \% \pm 7.7 \%$ on the second violation): participants rapidly learned to expect violations and seemed to adopt a more cautious response strategy. Accordingly, response times became slower after the first violation trial, and remained at this level throughout the experiment, for both violations and nonviolations (mean RT across trials $324 \pm 19$ ms until first violation, versus $378 \pm 5 \mathrm{~ms}$ after the first 
violation). Eventually, participants from the $75 \%$ violations group, who received a majority of violation trials, reached near-perfect performance in both violations and non-violations (mean per-participant accuracy from sixth trial: $95.8 \% \pm 2.2 \%$ for non-violations, versus $97.5 \% \pm 1.0 \%$ for violations).

The differences between principles were greatest on the first violation trial: participants made more mistakes or responded correctly more slowly on the first violation when it was a continuity violation (mean accuracy $44.4 \% \pm 14 \%$, mean correct RT $520 \pm 49 \mathrm{~ms}$ ) than when it was a solidity violation (mean accuracy $72.0 \% \pm 13 \%$, mean correct RT $486 \pm 82 \mathrm{~ms}$ ). In the following trials, as participants became more cautious, the effect on accuracy was much reduced (continuity violations $91.0 \% \pm 3.1 \%$; solidity violations $93.7 \% \pm 2.6 \%$ ), and the effect on response time disappeared (continuity violations $381 \pm 12 \mathrm{~ms}$; solidity violations $385 \pm 11 \mathrm{~ms}$ ).

\section{Debriefing}

As evidenced from post-experiment debriefing, participants were more likely to report having noticed the continuity than the solidity violations $\left(90.5 \%\right.$ vs. $78.3 \%, \mathrm{X}^{2}(1)=4.92$, $p=0.027)$, though the majority (74.5\% participants) noticed both types. Amongst those who recognized both types of violations, $24.5 \%$ judged both types of violations as equally surprising, while $21.7 \%$ found continuity violations more surprising and $28.3 \%$ found solidity violations more surprising, a non-significant difference $\left(\mathrm{X}^{2}(1)=0.924, p=0.336\right)$.

\section{Discussion}

In Experiment 1, participants performed a very simple task: reporting the location of an object quickly after it became visible. However, they sometimes erred when the location of the object did not conform to the basic physical principles of continuity and solidity, indicating that they spontaneously anticipated the position of the car in ways that conformed to those principles. 
Crucially, these errors were more frequent in the case of violations of continuity than for violations of solidity, in line with our hypothesis that solidity violations are not perceived as instances of continuity violations. Consistent with this result, the response time difference between non-violations and violations was larger for continuity than for solidity, and participants receiving a continuity event as the first violation were slower to produce a correct response than participants receiving a solidity event as the first violation.

Seeing unexpected events such as violations of core principles disrupts cognition in adults (Shurgin \& Flombaum, 2017) and infants (Cheries, Mitroff, Wynn, \& Scholl, 2008; Huntley-Fenner, Carey, \& Solimando, 2002) but may lead to increased learning, as has been shown in infants (Stahl \& Feigenson, 2015, 2017). Here, participants were able to learn to inhibit reflexive responses and instead adopted a slower and more cautious response strategy across all conditions. Once participants adopted the more cautious strategy, they performed at near ceiling accuracy when confronted with physical violations. The relative dampening of the effect of physical principle in the frequent (75\%) violation conditions is likely an effect of this strategy change. Thus, it seems that even though we found a clear difference in processing between continuity and solidity events, this difference is masked by a task-specific response strategy after only a few exposures. Interestingly, infants do not learn to expect violations of continuity after repeated exposures (Newcombe, Sluzenski \& Huttenlocher 2005), suggesting that the pattern we see in adults is not an effect of visual learning but rather of a metacognitive strategy.

Although Experiment 1 provided evidence that continuity and solidity are processed as two separate principles, two alternative explanations remain possible. First, in continuity violations, the object was seemingly displaced to an earlier position in its trajectory (Figure 3b), whereas in solidity violations, the end position of the object was seemingly displaced further 
along on its trajectory (Figure 3a). It is possible that these trajectory reversals imposed a cost on participants' attention, unrelated to the nature of the violation. Secondly, there was a difference in complexity between the continuity and solidity displays due to the presence of an additional solid block in solidity videos. Experiment 2 controlled for these factors.

\section{Experiment 2}

In Experiment 1, we had contrasted performance on solidity events with continuity events that displayed a different motion profile: continuity violations corresponded to a reversal of the car trajectory (as in Experiment 1, Figure 3a). Here we instead contrasted solidity violations with continuity violations whose motion profiles perfectly matched those in the solidity violations. In these events the car stopped behind the first screen, but appeared further along behind the second screen in violation trials (Figure 6b, continuity forward events). Thus they differ from the continuity trials featured in Experiment 1, henceforth referred to as continuity backward trials. Secondly, we matched the complexity of the visual display between the continuity and solidity events by adding a (non-active) solid barrier in the display (see Figure 6 below). Finally, since the difference in performance between continuity and solidity vanished quickly after the first violation trial in Experiment 1, in Experiment 2 we presented only one violation trial to each participant.

\section{Method}

Experiment 2 was identical to Experiment 1, except for the differences given here.

\section{Participants}

A sample size of 260 was determined via a power analysis based on the effect size from a pilot study (logistic regression with binomial distribution, two groups, odds ratio $=2.12$, $\operatorname{Pr}(\mathrm{Y}=1 \mid \mathrm{X}=1) \mathrm{H} 0=.25, \alpha=.05$, power $=80 \%)$. We then recruited participants online via 
Amazon Mechanical Turk, until we reached that sample size, after applying pre-defined exclusion criteria. Fourty-six participants $(14 \%)$ were excluded because they did not follow instructions, encountered technical issues or took the experiment several times (see Table S1). Furthermore, 40 trials $(2.9 \%)$ were excluded from analysis because the participant did not answer in the requested timeframe or because of other issues (see Table S3). In the final sample of 274 participants, ages ranged from 19 to 76 years $($ mean $=33.5), 125(46 \%)$ were women and $234(85 \%)$ were right-handed.

\section{Stimuli}

To equate visual complexity, a block similar to that of the solidity display was added to the continuity display, to the side opposite to the car's trajectory. In addition, the car was changed to a symmetric shape, so the outcome images with the car revealed did not differ between leftward and rightward stimuli. As a result, participants could no longer know just by looking at the outcome images if there had been a solidity violation. Finally, video timing was sped up, to further encourage fast answers based on expectations. Videos for Experiment 2 are available online at https://goo.gl/dBF8Vy. 
a. Solidity

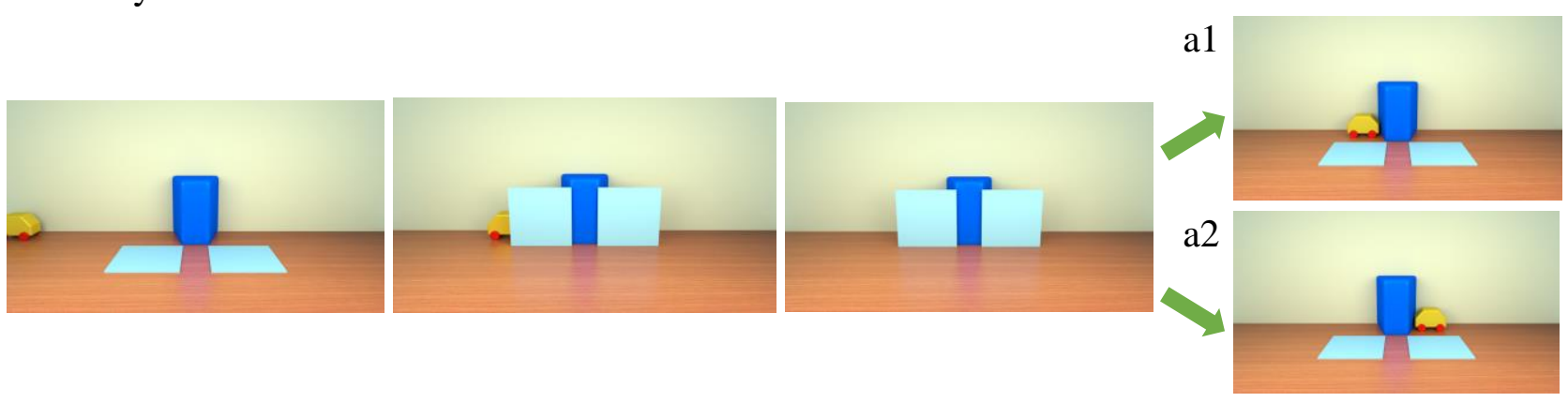

b. Continuity forward
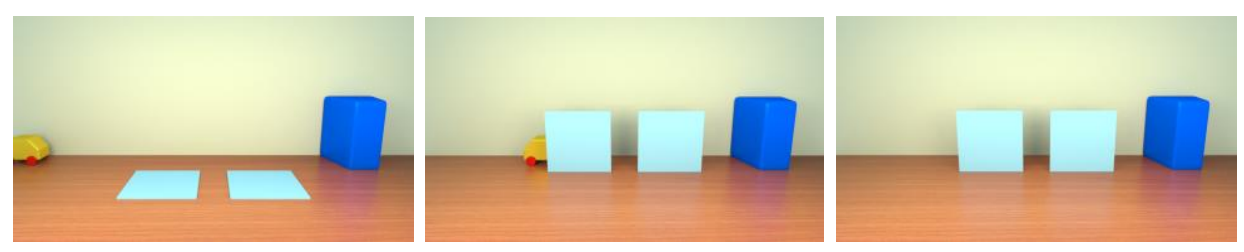

b1

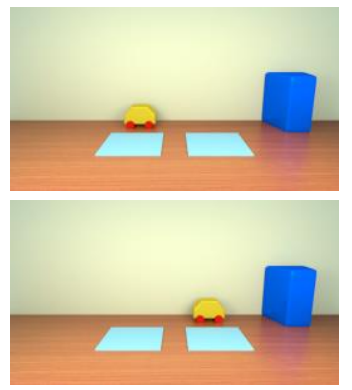

c. Continuity backward
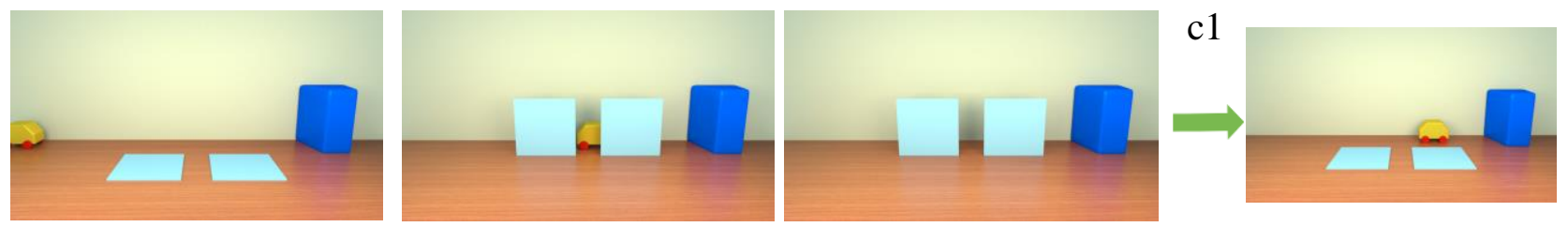

Figure 6. Structure of the five conditions from experiment 2, of which three are nonviolation conditions (a1-c1) and two are violation conditions (a2, b2). Forward and backward here refers to the displacement of the car during a violation, to distinguish the two types of continuity trials. Continuity backward (c) existed only in its non-violation version (c1) in this experiment, as this type of trial were used for the training and familiarization phases only.

\section{Procedure}

In the experiment, there was first a training phase and then a test phase.

During the training phase, participants saw four non-violation events. Half the participants received four continuity non-violation trials (two "forward" trials where the car 
stopped behind the first occluder, and two "backward" trials where it stopped behind the second occluder). The other half received two continuity "backward" non-violation trials (car stopping behind the second occluder) and two solidity non-violation trials (where the car stopped behind the first occluder).

The test phase, which immediately followed the training phase, was composed of five trials: four non-violations (familiarization trials) followed by one violation. For each of the training conditions (see above), half of the participants appeared in a continuity test condition and the other half in a solidity test condition. Participants in the continuity condition saw a single continuity 'forward' violation, preceded by four continuity non-violations events, two stopping behind the first screen (one from each direction: left to right and right to left) and two stopping behind the second screen (again in each direction). Participants in the solidity condition saw a single solidity violation, preceded by two solidity non-violations (in which the car stopped behind the first occluder; again one in each direction), and two continuity non-violations where the car stopped behind the second occluder. ${ }^{1}$ That way, the car's motion in the violation event was exactly the same in the continuity and solidity conditions (it ended its course behind the nearest occluder), and it was balanced in non-violations (half near side and half far side). As there was only one violation trial per participant, for this trial the side of the screen on which the car started (and thus its initial direction of motion) could not be counterbalanced within participants, so it was counterbalanced across participants.

\section{Results}

Data and analysis scripts are available online at https://osf.io/9daxp/.

\footnotetext{
${ }^{1}$ We were careful in the familiarizations to ensure that participants had been exposed to outcomes involving both possible locations, as pilot data had shown that repeated and multiple exposures to only single outcome location led to likely strategic responses on the part of participants.
} 
The two training conditions gave similar results, so we analyzed the data with this variable collapsed. Collapsing across principles, the accuracy on the violation trial $(42.7 \%$ $\pm 5.9 \%$ ) was much lower than on the first non-violation trial from the same principle $(97.1 \% \pm 2.0 \%)$ or the mean of all non-violation trials $(98.0 \% \pm 1.0 \%)$.

Accuracy

Importantly for our hypothesis (and mirroring the results of Experiment 1) participants were less accurate for continuity violations than for solidity violations. Pre-planned analyses revealed that accuracy on the violation trials was significantly lower in the continuity condition $(33.8 \% \pm 8.0 \%)$ than in the solidity condition $(51.9 \% \pm 8.5 \%), \mathrm{z}=3.0, p=.003$ (logistic regression with principle as independent variable, effect size in Logit space $=0.75[0.26,1.24]$, standard error $=0.25)$. On the non-violation trials, accuracy was virtually identical across principles $(97.1 \% \pm 2.0 \%$ for continuity forward versus $97.0 \% \pm 2.3 \%$ for solidity) and thus did not differ significantly (ANOVA over condition means; $F(1,272)=0.003, p>.250, \eta^{2}<.001$, 95\% CI $[0, .004])$. 
Experiment 2 - Accuracy

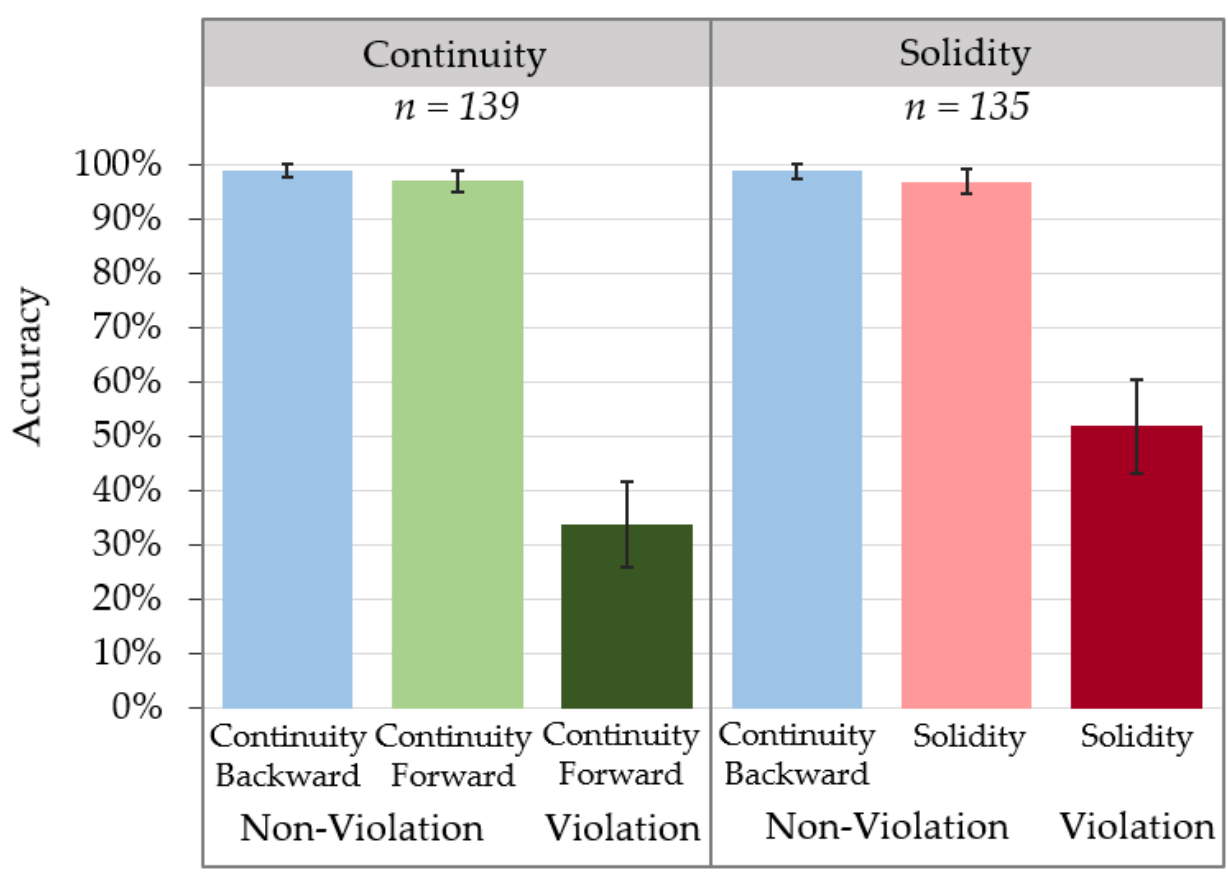

Figure 7. Experiment 2 mean accuracies, depending on Group (facet), Principle and

Violation (abscissa and color). Error-bars represent 95\% confidence intervals.

\section{Response times}

We also analysed response times on correct answers (see Figure 8), after excluding eight outliers defined as RT $>3 *$ mad (median absolute deviation) from the group median and 157 participants who lacked correct answers on some type of condition (typically the violation condition). RTs showed a conceptually similar trend as error rates, with a longer response time for correct answers on continuity (forward) violations than solidity violations ( $563 \pm 81 \mathrm{~ms}$ vs $449 \pm 56 \mathrm{~ms}, F(1,107)=5.8, p=.018, \eta^{2}{ }_{p}=0.051,95 \%$ CI $\left.[0.001, .15]\right)$. Response times on the non-violation trials did not differ significantly (345 $\pm 45 \mathrm{~ms}$ for continuity forward vs $336 \pm 26 \mathrm{~ms}$ 
for solidity, $F(1,107)=0.17, p>.250, \eta^{2}{ }_{p}=0.002,95 \%$ CI $\left.[0,0.047]\right)$.

\section{Experiment 2 - Response Time on Correct Answers}

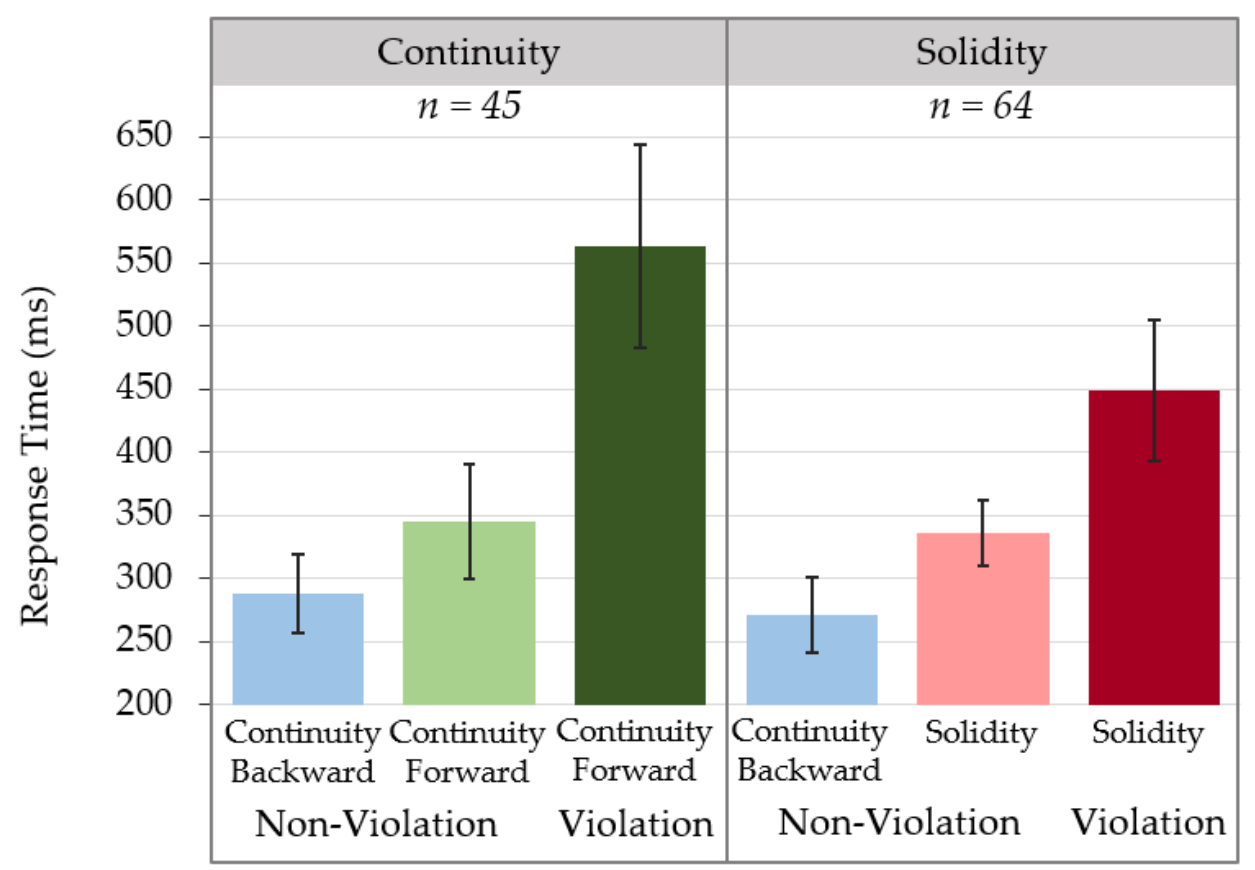

Figure 8. Experiment 2 mean response times (means of individual means on correct answers, including only participants with at least a correct answer in all conditions), depending on Group (facet), Principle and Violation (abscissa and color). Error-bars represent 95\% confidence intervals.

\section{Debriefing}

As evidenced from post-experiment debriefing, $70 \%$ of participants reported having noticed the car doing something physically impossible. This proportion did not differ between continuity and solidity violations $\left(67 \%\right.$ vs. $\left.73 \%, \mathrm{X}^{2}(1)=1.06, p>.250\right) .97 \%$ of the participants who were exposed to and noticed a continuity violation agreed that 'the car popped into and out of existence'. $91 \%$ of the participants who were exposed to a solidity violation and noticed it agreed with the interpretation of "the car going through the solid block", but $39 \%$ also agreed that "the car appeared in a location that it should not have appeared in, by "popping out and into existence"". 


\section{Discussion}

In Experiment 2, we again contrasted continuity vs. solidity violations, but this time equating the trajectory of the object and visual complexity across conditions. Again, we found that continuity violations more severely disrupt RT's and accuracy than solidity violations in the object location task, in line with distinct representations of these principles and a stronger influence of the continuity principle. Experiment 2 thus demonstrates that this effect is independent of low-level perceptual factors such as momentum in motion, or overall complexity of the display. The results of the post experiment debriefing suggest that performance differences in the object detection task cannot be attributed to differences in the explicit understanding of the continuity and solidity violations (as a similar number of participants detected the violation in both conditions). Moreover, in contrast to Experiment 1 we were able to unconfound the movement pattern from the physical principle in the violation trials. Given that object motion and displacement patterns were perfectly matched across the solidity and continuity conditions, this factor cannot explain our results.

However, in controlling for the low-level motion patterns a new confound was introduced in the familiarization sets of Experiment 2. Participants who perceived a continuity forward violation at test were familiarized with two continuity backward and two continuity forward nonviolations. Participants who perceived a solidity violation at test were familiarized with two continuity backward and two solidity non-violations. Thus, in the continuity condition, participants had only seen continuity videos during familiarization, whereas in the solidity condition, participants had seen both continuity and solidity videos during familiarization. This might have influenced the results, as participants in the continuity condition received relatively 
more training with the relevant non-violation condition ${ }^{2}$. Experiment 3 was designed to overcome this issue.

\section{Experiment 3}

Experiment 3 was a close replication of Experiment 2, with two further goals. First, we directly tested for the influence of object motion pattern at test, by contrasting continuity forward and continuity backward test events (in addition to solidity), in order to more clearly isolate and assess the role of motion and displacement patterns. Secondly, we asked whether altering the ratios of continuity vs. solidity events during training and familiarization would have any effect on responses to identical event types at test. If not, then we can discount this as a possible explanatory factor for the results of Experiment 2 above.

\section{Method}

Experiment 3 was again a between-participant design with each participant appearing in only one of the four conditions. For rhetorical clarity, Experiment 3 is explained here as two subexperiments with two conditions each, even though it was conducted and analyzed as a single experiment with four conditions.

Experiment 3a was designed to compare continuity and solidity with a matched set of familiarization trials. Thus all participants received two (non-violation) continuity backward trials and two (non-violation) solidity forward trials for each of the training and familiarization phases (a total of four trials in each). Then they received one test (violation) trial, which depended on the experimental condition: the solidity group received a solidity (forward) violation, and the continuity group received a continuity backward violation. Consequently, the amount of non-violation trials of each kind were kept the same in both conditions, as to avoid

\footnotetext{
${ }^{2}$ We thank an anonymous reviewer for pointing out this confound to us.
} 
confounding the training and familiarization sets with physical principle (the continuity vs. solidity manipulation). These conditions instead confounded the car's motion pattern (during the violation trials) with physical principle (similarly to Experiment 1), as continuity backward was contrasted with solidity. This issue is dealt with in the design of $3 \mathrm{~b}$.

Experiment $3 \mathrm{~b}$ was designed to isolate motion pattern while keeping the physical principle constant. Training and familiarization thus included two continuity forward and two continuity backward trials each, and the test trials were either one continuity forward trial or one continuity backward trial (depending on experimental condition). This allowed us to directly assess the impact of motion pattern (for continuity events at violation). Moreover, by comparing the continuity backward violation events in Experiments $3 \mathrm{a}$ vs. 3b, we were able to directly assess any possible influence of categories of event types in the familiarization sets (where Experiment 3a involved continuity and solidity during familiarization and training while Experiment $3 b$ involved only continuity events). Finally, the interaction between familiarization set (continuity and solidity vs. only continuity) and motion pattern (forward vs. backward) dissociates the influence of physical principle from that of the motion pattern (and thus direction of displacement during violation events, see Table 1 below).

The procedures differed in two additional ways from Experiment 2: the training trials that were presented before the familiarization trials had answer cues (icons indicating the correct response) and a larger response window; and analyses included only participants who made no more than a single error on familiarization trials. These two changes were implemented in order to ensure that the stimuli were attended to and familiarized with to a similar extent in all conditions, thus further constraining the amount of experience with each type of display that each participant receives. 


\section{Participants}

A power analysis $(\alpha=.05$, power $=80 \%)$ based on the observed accuracy effect size from Experiment 2 suggested 132 participants per group for simple effects, a number that was increased by a factor 1.5 in order to allow consideration of interaction effects. A total of 1231 participants were recruited via Mechanical Turk. A total of $436(35.4 \%)$ participants were excluded based on pre-defined exclusion criteria: because they did not follow instructions, failed to respond within the time window, or made more than one error during familiarization trials (see Table S2). The final sample consisted of 795 participants of ages ranging between 18 and 81 years $($ mean $=35.8)$, of which 463 were female and 696 were right-handed.

\section{Stimuli}

Videos featured the symmetric car (as in Experiment 2). The solid block was only present in solidity trials, whereas continuity (forward as well as backward) trials featured a blue square matched in color and size with the solid block. The block and the square were both presented in the middle of the screen between the two occluders, as in Experiment 1. Unlike Experiments 1 and 2, the car started moving at the same time in all conditions, rendering "backward" videos slightly longer than "forward" videos. In the end a debriefing question was presented: "Did you ever notice the car appearing to have moved in a physically impossible way?" (it had thus a slightly different wording compared to Experiments 1 and 2), followed by two control questions (one about whether the car ever appeared to have been replaced, and one about whether any flickering was seen during animations). Example videos for each condition are available online: https://tinyurl.com/y56fpnm5

\section{Procedure}


The stimuli were presented online using the jsPsych javascript library (version 6.0.2, de Leeuw, 2015). Participants in all conditions saw four training trials followed by a pause, then four familiarization trials, all of the non-violation kind. The $9^{\text {th }}$ trial was a violation trial, just as in Experiment 2. The training block and the familiarization block contained the same four videos in random order, except that the training trials had more time to respond and featured answer cues (icons representing the keys on the keyboard that the participant was supposed to press, always conveying the correct response). The contents of the trials further differed depending on condition: in Experiment 3a all participants saw two solidity (forward) trials and two continuity backward trials in each of the training and familiarization blocks. They then saw different violation trials depending on condition: participants in the solidity condition saw a solidity violation trial, and participants in the continuity condition saw a continuity (backward) violation trial. Thus, the confound in the training set that was present in Experiment 2 is not present here: both groups have seen the same amount of continuity and solidity displays at the moment when the violation trial started.

Participants in Experiment $3 \mathrm{~b}$ saw instead two continuity forward trials and two continuity backward trials in each of the training and familiarization blocks, followed by either a continuity forward or a continuity backward violation trial (depending on condition). This allowed us to assess the impact of forward and backward displacement on violation sensitivity while keeping physical principle constant (continuity only), thus controlling for the motion confound that remained in Experiment $3 a$.

Together, Experiments $3 \mathrm{a}$ and $3 \mathrm{~b}$ allowed us to dissociate possible effects of forward vs. backward displacements (relative to the car's trajectory) from possible effects of solidity vs. continuity, while still either controlling for familiarization with each type of display (as in 
Experiment 3a) or in testing its impact directly (in contrasting performance on continuity backward trials for $3 a$ vs. 3b). Whereas Experiment $3 a$ tests continuity against solidity in the presence of a motion pattern confound, Experiment $3 \mathrm{~b}$ allows a direct assessment of the magnitude of any effect of motion pattern. See Table 1 for the full design.

Table 1. The conditions of Experiments $3 \mathrm{a}$ and $3 \mathrm{~b}$. The columns under "Experiment timeline" shows the phases of the experiment, and the columns under "Statistical comparisons" show how the different conditions are analyzed in terms of simple and interaction effects. Simple effects reflect the impact of motion pattern (3b) and motion pattern and principle taken together (3a), whereas their interaction reflects the conceptual subtraction of $3 b$ from $3 a$, leaving the impact of physical principle isolated.

Experiment timeline

Statistical comparisons

\begin{tabular}{|c|c|c|c|c|}
\hline & \multicolumn{2}{|c|}{ Experiment timeline } & \multicolumn{2}{|c|}{ Statistical comparisons } \\
\hline & $\begin{array}{l}\text { Training +familiarization } \\
\text { (non-violation) trials }(x 4)\end{array}$ & violation trial & Simple effects & Interaction effect \\
\hline \multirow{2}{*}{$3 \mathrm{a}$} & \multirow{2}{*}{$\begin{array}{l}\text { Solidity }+ \\
\text { Cont. Backward }\end{array}$} & $\begin{array}{l}\text { Solidity } \\
\text { (forward) }\end{array}$ & \multirow{2}{*}{$\begin{array}{l}\text { Motion pattern } \\
\text { + continuity vs. } \\
\text { Solidity }\end{array}$} & \multirow{4}{*}{$\begin{array}{l}\text { Continuity vs. } \\
\text { Solidity } \\
\text { (controlling for } \\
\text { motion pattern) }\end{array}$} \\
\hline & & $\begin{array}{l}\text { Continuity } \\
\text { backward }\end{array}$ & & \\
\hline \multirow{2}{*}{$3 b$} & \multirow{2}{*}{$\begin{array}{l}\text { Cont. forward + } \\
\text { Cont. Backward }\end{array}$} & $\begin{array}{l}\text { Continuity } \\
\text { forward }\end{array}$ & \multirow[t]{2}{*}{ Motion pattern } & \\
\hline & & $\begin{array}{l}\text { Continuity } \\
\text { backward }\end{array}$ & & \\
\hline
\end{tabular}

\section{Results}

Data and analysis scripts are available online at https://osf.io/9daxp/.

\section{Accuracy}

As participants were allowed to make no more than one error on familiarization (nonviolation) trials in order to be included in the analysis, the following analyses focuses on the participants' performance on the single violation trial. Importantly for dissociating our two 
factors, familiarization set (Exp. 3a vs 3b) interacted with the motion pattern (Forward vs. Backward); $z=2.01, p=.037, B=.643,95 \%$ CI $[.039,1.25]$. In other words, the relative processing advantage of solidity over continuity backward in Experiment $3 \mathrm{a}(z=7.42, p<.0001$, $B=1.64,95 \%$ CI $[1.21,2.08])$ was significantly stronger than the advantage of continuity forward over continuity backward in Experiment $3 b(z=4.62, p<.0001, B=.994,95 \% \mathrm{CI}$ $[.576,1.42])$. This suggests a role of physical principle over and above that of simple motion and displacement patterns of the object. Moreover, given that we had controlled for the number of continuity vs. solidity trials in training and familiarization in Experiment 3a, any difference in accuracy between continuity and solidity here cannot be explained by differences in the training set.

Replicating the finding from Experiment 2, comparing only participants who received forward trials (i.e. solidity or continuity forward), accuracy for solidity $(68.3 \% \pm 6.5 \%$ correct) was significantly higher than for continuity forward $(46.7 \% \pm 7.0 \%$ correct $) ; z=4.33, p<.0001$, $B=.899,95 \%$ CI $[.495,1.31]$. Accuracy was thus significantly higher for solidity trials than for its closest competitor among continuity trials (continuity forward). Backward trials did not show a significant difference depending on training set; $z=1.13, p=.260, B=.257,95 \%$ CI [-.189, .704]. This suggests that more exposure with the continuity principle in the training and familiarization phases of Experiment 3b compared to Experiment 3a did not affect the accuracy of responses.

\section{Response times}

A conceptually similar trend was observed for response times among the participants who responded accurately $(\mathrm{N}=309$, after excluding 28 statistical outliers defined by $\mathrm{RT}>3 * \mathrm{mad})$. These results do not offer any particular reason to believe that a speed-accuracy trade-off is 
responsible for our result. The interaction between familiarization set and motion pattern was not significant $\left(F(1,305)=1.19, p=.275, \eta^{2} \mathrm{p}=.004,95 \%\right.$ CI $\left.[0, .029]\right)$, however, the numerical trends agreed with those for accuracy: Participants in Experiment 3a seeing a solidity violation responded on average after $410 \mathrm{~ms} \pm 13 \mathrm{~ms}(\mathrm{n}=122)$, faster than any of the continuity conditions ( 3 a continuity backward: $478 \mathrm{~ms} \pm 32 \mathrm{~ms}, \mathrm{n}=49 ; 3 \mathrm{~b}$ continuity forward: $507 \mathrm{~ms} \pm 22 \mathrm{~ms}, \mathrm{n}=89 ; 3 \mathrm{~b}$ continuity backward: $548 \mathrm{~ms} \pm 36 \mathrm{~ms}, \mathrm{n}=49$ ). Solidity thus yielded significantly faster correct responses than its closest competitor among continuity trials (3a continuity backward), $F(1,169)$ $=22.4, p<.0001, \eta_{p}^{2}=.117,95 \%$ CI $[.041, .210]$; and faster than continuity forward, $F(1,209)$ $=62.0, p<.0001, \eta_{p}^{2}=.229,95 \%$ CI $[136, .320]$. The response time difference between the two conditions in experiment $3 b$ was also significant $\left(F(1,136)=4.22, p=.042, \eta^{2} p=.030,95 \% \mathrm{CI}\right.$ $[0, .105])$. Unlike the pattern observed for accuracy, among participants receiving continuity backward trials as test those who received only continuity trials ( $3 b)$ were significantly slower $\left(F(1,96)=8.67, p=.004, \eta^{2} \mathrm{p}=. .083,95 \% \mathrm{CI}[.009, .199]\right)$. See figure 9 for an overview of the results. 


\section{Experiment 3}

\section{(violation trials)}

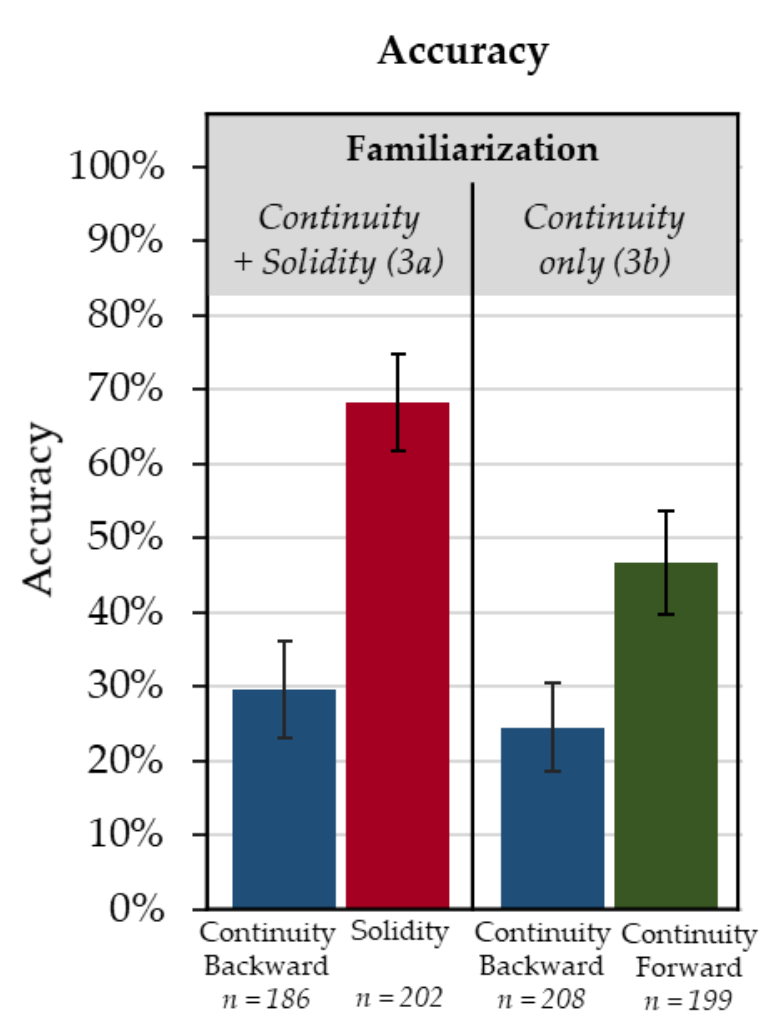

\section{Response Time \\ (correct answers)}

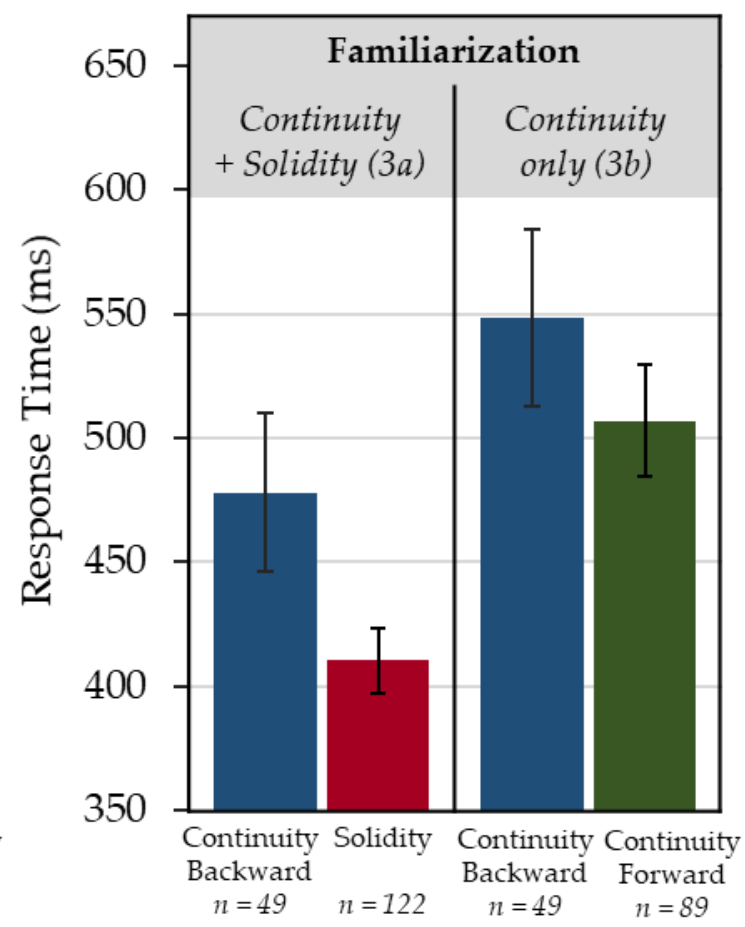

Figure 9. Accuracy and response times for violation trials in Experiment 3. Solidity trials yield faster and more accurate responses than any type of continuity trial. Error-bars represent $95 \%$ confidence intervals.

\section{Debriefing}

Finally, debriefing showed that $51.6 \% \pm 3.5 \%$ of participants noticed the car moving in a physically impossible way. This proportion differed between conditions: participants in the solidity condition (Experiment 3a) were least likely to detect the violation $(38.1 \% \pm 6.8 \%$ ), followed by continuity forward (Experiment $3 \mathrm{~b}, 51.8 \% \pm 7.0 \%$ ) and finally continuity backward (Experiment 3a: 53.2\% $07.2 \%$, Experiment 3b 63.0\% $\pm 6.6 \%$ ). A logistic regression with explicit violation detection as dependent variable revealed a significant main effect of motion pattern $(z=$ $2.28, p=.022, B=.461,95 \%$ CI $[.067, .858])$ and a borderline non-significant main effect of familiarization set $(z=1.96, p=.0503, B=.402,95 \%$ CI $[.000, .806])$. The interaction was not 
significant $(z=.529, p=.597)$. The explicit detection disadvantage of solidity compared to its closest competitor, continuity forward, was also significant: $\mathrm{z}=2.73, \mathrm{p}=.006, B=.555,95 \% \mathrm{CI}$ $[.16, .95]$. As this result appeared to differ compared to the explicit detection question in Experiment 2, we performed a logistic regression including the data from Experiment 2 and the two closest matching conditions from Experiment 3 (continuity forward and solidity). This analysis revealed a main effect of Experiment: participants in solidity and continuity forward conditions in Experiment 3 was generally less likely to explicitly notice a violation, compared to the participants in Experiment $2(44.9 \%$ vs. $70.1 \% ; \mathrm{z}=2.76, \mathrm{p}=.006, \mathrm{~B}=.63,95 \% \mathrm{CI}[.188$, 1.09]). Furthermore, an interaction effect was revealed between Experiment and Group $(\mathrm{z}=2.58$, $\mathrm{p}=.01, \mathrm{~B}=.863,95 \% \mathrm{CI}[.206,1.52])$. The difference in wording and presentation of this question between Experiment 2 and Experiment 3 may be responsible for these differences: In Experiment 2 (and 1) we asked "Did you ever notice that the car did something physically impossible? If so, what did it do?", whereas in Experiment 3 the question read "Did you ever notice the car appearing to have moved in a physically impossible way?",

\section{Non-violation trials}

As we included only participants making not more than one error on familiarization trials when analyzing the violation trials, a matched analysis of familiarization (non-violation) trial performance was not meaningful. In order to rule out the possibility that the observed solidityadvantage for violation trials was due to the presence of the solid block making location judgments somehow easier in general, we inspected familiarization trial performance among all participants who satisfied all other inclusion criteria $(\mathrm{N}=910)$. Accuracy for familiarization trials was the lowest for solidity trials $(93.4 \% \pm 1.9 \%)$, lower than both continuity forward trials 
$(95.7 \% \pm 1.6 \%)$ and matched continuity backward trials from the same sub-experiment (3a;

$98.1 \% \pm 1.1 \%$ ), suggesting against this possible worry.

\section{Discussion}

In Experiment 3 we again showed that violations of continuity decreased the accuracy of object detection compared to violations of solidity. We found an additional advantage of forward compared to backward displacement of the car, however this could not account for the accuracy difference between continuity and solidity. Comparing trials with forward displacement only (solidity vs. continuity forward) we still found a significant advantage for solidity on violation trials, for both accuracy and response times, thus replicating the results from Experiment 2. Comparing continuity trials across familiarization sets allowed us to test a possible worry that the difference between solidity and continuity violations in Experiment 2 was due to the solidity participants receiving fewer solidity familiarization trials than continuity participants received continuity familiarization trials. This possible confound seems not to have impacted detection accuracy, as accuracy was similar for the two continuity backward participant groups receiving different numbers of continuity familiarization trials. Moreover, results for response times pointed essentially in the same direction as those for accuracy. Whereas not significant, this numerical pattern suggests no reason to suggest that a speed-accuracy trade-off produced our result. Our results also elucidate the role of the ratios of continuity vs. solidity trials in the training and familiarization phases, by eliminating any difference between these in Experiment 3a. The fact that we still found significant differences between continuity and solidity in this condition further suggests that this confounding factor from Experiment 2 could not explain the main results. 


\section{General discussion}

In the present study, we established a method for investigating sensitivity to violations of core physical principles in adults. Across three experiments, we find evidence that violations of the principles of continuity and solidity can disrupt adult's abilities to respond to the location of an object, that representations of these principles seem distinct, and furthermore that violations of continuity are more disruptive than violations of solidity. Our findings cannot be attributed to low-level differences such as display complexity, motion patterns, or patterns of apparent displacement for objects that undergo violations. The results cannot be attributed to participants having a weak understanding of solidity in the displays as most participants agreed with the interpretation that the car went through the solid block (in Experiments 1 and 2), and (in Experiment 1) they reported equal explicit surprise to both types of violations.

On a priori grounds, solidity could have been considered as a subcase of continuity: violations of solidity, where two objects traverse each other, can also be construed as violations of continuity, where an object vanishes and then comes back to existence on the other side (see Figure 1). Considerations of theoretical parsimony thus originally made theories where the two principles are merged more appealing (Spelke, 1994). Our findings contradict this idea. If solidity violations were processed as continuity violations, both should induce the same level of error and processing delays. If solidity violations were viewed as violating both solidity and continuity, if anything they should induce even higher expectations than simple continuity violation. In contrast, in our task we found that solidity violations were less disruptive than continuity violations.

Functional differences between continuity and solidity? 
It has long been claimed that the continuity principle is fundamental for solving the problem of object individuation over time (Pylyshyn, 1989; 2000; 2001). As we experience the world, there is a basic problem of computing whether an object experienced at time $\mathrm{T} 1$ is the same individual as an object experienced at time T2. One way to resolve this problem is to assess whether the object could have travelled from a location L1 at time T1 to a location L2 and time $\mathrm{T} 2$ given a prior on that object's speed and trajectory. If not, the object experienced at L1/T1 and that at L2/T2 is not treated as the same individual. A wealth of literature focusing on adult perception demonstrates that the visual system indeed uses object continuity to track and individuate objects dynamically through visual noise and occlusion (Flombaum \& Scholl, 2006, Yi et al., 2008), suggesting that the continuity principle is indeed fundamental to object tracking. This model, however, is silent on how and whether solidity constraints are involved. It is thus an open empirical question as to whether solidity would participate in these fundamental object individuation processes. Our results suggest that this is not (or at least less) the case, as continuity violations produce systematically far more errors in response to detecting object locations compared to solidity violations.

The present results are thus consistent with object tracking mechanisms that place a higher priority on objects' visible trajectories than on solidity constraints regarding where the objects can travel. If the car's speed, trajectory, and visibility are all that matters, then participants in the solidity conditions in our experiments would anticipate the car to appear on the next visible location in its path, which is right next to the second occluder (behind which the car is revealed moments later). In contrast, in the continuity conditions the car would first be expected to be seen between the occluders (twice in the case of continuity backward), and when 
it does not appear there it is assumed to have stopped behind the occluder where it was last seen to enter.

Experiment 3 revealed an additional effect of forward compared to backward displacement in continuity violations: forward displacements gave rise to higher accuracy and faster responses compared to backward displacements. One explanation, in line with the one provided above, could be that object-tracking mechanisms take into account the fallibility of visual attention. In the continuity backward case participants have access to positive evidence that the car has passed the space in the center, so provided that this part of the event was noticed, the car cannot be anywhere else than behind the second occluder. In contrast, in the continuity forward case, participants have only negative evidence that the car did not pass between the occluders - either it did not pass, or it passed but avoided the participant's attention, increasing uncertainty of its location. In the solidity case, however, the option to see the car pass is simply not there, but rather the solid block should imply a hard constraint on the possibility of the car passing through this location. Nevertheless, we find that this constraint does not seem to have a strong impact. A mechanism sensitive to speed and trajectory but not to physical barriers thus explains our full pattern of results.

\section{Limitations}

Finally, we would qualify here that our study focused on spatial continuity and spatial consequences of solidity constraints on object motion. We have not investigated other types of continuity, for example continuity of features such as shape or color (featural continuity). Whereas featural and spatial continuity appear to be dissociated in infants (Xu \& Carey, 1996) but have been shown to combine as cues to object constancy in adults (see Shurgin \& 
Flombaum, 2017), our data do not speak to whether these different forms of continuity are implemented in a single mechanism. This would be a topic for future investigation.

\section{Author Note}

Brent Strickland developed the study concept. All authors contributed to the study design. Stimuli creation, programming, data collection and processing were performed by Ghislaine Labouret (Experiments 1 and 2) and Andreas Falck (Experiment 3). Andreas Falck, Ghislaine Labouret, Brent Strickland and Véronique Izard performed the data analysis and interpretation. Ghislaine Labouret drafted the first version of the manuscript, Andreas Falck prepared the final version. Brent Strickland, Véronique Izard, Frank Keil, and Annie Wertz provided critical revisions. All authors approved the final version of the manuscript for submission. Part of the data presented in this manuscript were presented as a poster at the Vision Sciences Society 2015 meeting (Strickland, Wertz, Labouret, Keil \& Izard; 2015). Data, analysis scripts, stimuli, and supplemental material is available online at https://osf.io/9daxp/.

Correspondence concerning this article should be addressed to either Andreas Falck or Brent Strickland, Institut Jean Nicod, Département d'études cognitives, ENS, EHESS, CNRS, PSL University, UMR 8129, 29 Rue d’Ulm, 75005 Paris, France. E-mail: falck.andreas@gmail.com or stricklandbrent@gmail.com.

\section{Acknowledgments}

Département d'Etudes Cognitives receives support from Agence Nationale de la Recherche (grants ANR-10-IDEX-0001-02 PSL and ANR-17-EURE-0017 FrontCog). Andreas Falck gratefully acknowledges support from the Swedish Research Council, grant no. 2016-06783. Brent Strickland gratefully acknowledges support from PSL University (Aux Frontieres des Labex), grant IPFBW 2016-151. This study contributes to the IdEx Université de Paris ANR-18- 
IDEX-0001. Furthermore, this research received funding from the European Research Council (ERC) via two grants awarded to Philippe Schlenker: under the European Union's Seventh Framework Programme (FP/2007-2013)/ERC (grant agreement no. 324115, FRONTSEM); and under the European Union's Horizon 2020 research and innovation programme (grant agreement no. 788077 , Orisem). 


\section{References}

Baillargeon, R. (1986). Representing the existence and the location of hidden objects: Object permanence in 6- and 8-month-old infants. Cognition, 23(1), 21-41. https://doi.org/10.1016/0010-0277(86)90052-1

Baillargeon, R. (1987). Object permanence in 31/2- and 41/2-month-old infants. Developmental Psychology, 23(5), 655-664. https://doi.org/10.1037/0012-1649.23.5.655

Baillargeon, R. (2004). Infants' Physical World. Current Directions in Psychological Science, 13(3), 89-94. https://doi.org/10.1111/j.0963-7214.2004.00281.x

Baillargeon, R. (2008). Innate Ideas Revisited: For a Principle of Persistence in Infants' Physical Reasoning. Perspectives on Psychological Science, 3(1), 2-13. https://doi.org/10.1111/j.1745-6916.2008.00056.x

Baillargeon, R., \& DeVos, J. (1991). Object Permanence in Young Infants: Further Evidence. Child Development, 62(6), 1227-1246. https://doi.org/10.1111/j.14678624.1991.tb01602.x

Cacchione, T., \& Amici, F. (2015). Cohesion as a Principle for Perceiving Objecthood: Does It Apply to Animate Agents? Swiss Journal of Psychology, 74(4), 217-228. https://doi.org/10.1024/1421-0185/a000164

Carey, S. (2011). Précis of The Origin of Concepts. Behavioral and Brain Sciences, 34(03), 113124. https://doi.org/10.1017/S0140525X10000919

Cheries, E. W., Mitroff, S. R., Wynn, K., \& Scholl, B. J. (2008). Cohesion as a constraint on object persistence in infancy. Developmental Science, 11(3), 427-432. https://doi.org/10.1111/j.1467-7687.2008.00687.x 
Cheries, E. W., Mitroff, S. R., Wynn, K., \& Scholl, B. J. (2009). Do the same principles constrain persisting object representations in infant cognition and adult perception? The cases of continuity and cohesion. In B. M. Hood \& L. R. Santos (Eds.), The Origins of Object Knowledge (pp. 107-134). Oxford University Press. Retrieved from http://doi.org/10.1093/acprof:oso/9780199216895.003.0005

de Leeuw, J. R. (2015). jsPsych: A JavaScript library for creating behavioral experiments in a web browser. Behavior Research Methods, 47(1), 1-12. doi:10.3758/s13428-014-0458-y.

Faul, F., Erdfelder, E., Lang, A.-G., \& Buchner, A. (2007). G*Power 3: A flexible statistical power analysis program for the social, behavioral, and biomedical sciences. Behavior Research Methods, 39(2), 175-191. https://doi.org/10.3758/BF03193146

Feigenson, L., Carey, S., \& Hauser, M. (2002). The Representations Underlying Infants' Choice of More: Object Files Versus Analog Magnitudes. Psychological Science, 13(2), 150156. https://doi.org/10.1111/1467-9280.00427

Flombaum, J. I., \& Scholl, B. J. (2006). A temporal same-object advantage in the tunnel effect: Facilitated change detection for persisting objects. Journal of Experimental Psychology: Human Perception and Performance, 32(4), 840-853. https://doi.org/10.1037/00961523.32 .4 .840

Hood, B. M., Carey, S., \& Prasada, S. (2000). Predicting the Outcomes of Physical Events: TwoYear-Olds Fail to Reveal Knowledge of Solidity and Support. Child Development, 71(6), 1540-1554. https://doi.org/10.1111/1467-8624.00247

Huntley-Fenner, G., Carey, S., \& Solimando, A. (2002). Objects are individuals but stuff doesn't count: Perceived rigidity and cohesiveness influence infants' representations of small 
groups of discrete entities. Cognition, 85(3), 203-221. https://doi.org/10.1016/S0010$\underline{0277(02) 00088-4}$

Keen, R. (2003). Representation of Objects and Events Why Do Infants Look So Smart and Toddlers Look So Dumb? Current Directions in Psychological Science, 12(3), 79-83. https://doi.org/10.1111/1467-8721.01234

Leslie, A. M. (1988). The necessity of illusion: Perception and thought in infancy. In L. Weiskrantz (Ed.), Thought without language (pp. 185-210). Oxford: Clarendon Press.

Mitroff, S. R., Scholl, B. J., \& Wynn, K. (2004). Divide and Conquer: How Object Files Adapt When a Persisting Object Splits Into Two. Psychological Science, 15(6), 420-425. https://doi.org/10.1111/j.0956-7976.2004.00695.x

Newcombe, N. S., Sluzenski, J., \& Huttenlocher, J. (2005). Preexisting Knowledge Versus OnLine Learning: What Do Young Infants Really Know About Spatial Location? Psychological Science, 16(3), 222-227. https://doi.org/10.1111/j.09567976.2005.00807.x

Pylyshyn, Z. W. (1989). The role of location indexes in spatial perception: a sketch of the FINST spatial index model. Cognition, 32, 65-97.

Pylyshyn, Z. W. (2000). Situating vision in the world. Trends in Cognitive Sciences, 4(5), 197207. https://doi.org/10.1016/S1364-6613(00)01477-7

Pylyshyn, Z. W. (2001). Visual indexes, preconceptual objects, and situated vision. Cognition, 80(1-2), 127-158. https://doi.org/10.1016/S0010-0277(00)00156-6

Qualtrics Labs, Inc. (2019). https://www.qualtrics.com [online experiment platform]. Provo, UT, USA. 
Santos, L. R. (2004). “Core Knowledges”: a dissociation between spatiotemporal knowledge and contact-mechanics in a non-human primate? Developmental Science, 7(2), 167-174. https://doi.org/10.1111/j.1467-7687.2004.00335.x

Scholl, B. J., \& Leslie, A. M. (1999). Explaining the infant's object concept: beyond the perception/cognition dichotomy. In E. Lepore \& Z. Pylyshyn (Eds.). What is Cognitive Science? (pp. 26-73). Oxford, UK: Blackwell.

Schurgin, M. W., \& Flombaum, J. I. (2017). Exploiting core knowledge for visual object recognition. Journal of Experimental Psychology: General, 146(3), 362-375. https://doi.org/10.1037/xge0000270

Spelke, E. S. (1990). Principles of Object Perception. Cognitive Science, 14(1), 29-56. https://doi.org/10.1207/s15516709cog1401_3

Spelke, E. S. (1994). Initial knowledge: six suggestions. Cognition, 50(1-3), 431-445. https://doi.org/10.1016/0010-0277(94)90039-6

Spelke, E. S., Breinlinger, K., Macomber, J., \& Jacobson, K. (1992). Origins of knowledge. Psychological Review, 99(4), 605-632. https://doi.org/10.1037/0033-295X.99.4.605

Stahl, A. E., \& Feigenson, L. (2015). Observing the unexpected enhances infants' learning and exploration. Science, 348(6230), 91-94. https://doi.org/10.1126/science.aaa3799

Stahl, A. E., \& Feigenson, L. (2017). Expectancy violations promote learning in young children. Cognition, 163, 1-14. https://doi.org/10.1016/j.cognition.2017.02.008

Strickland, B., \& Scholl, B. J. (2015). Visual perception involves event-type representations: The case of containment versus occlusion. Journal of Experimental Psychology. General, 144(3), 570-580. https://doi.org/10.1037/a0037750 
Strickland, Wertz, Labouret, Keil \& Izard (2015). The principles of object continuity and solidity in adult vision: Some discrepancies in performance. Poster presented at the annual meeting of the Vision Sciences Society, Saint Pete's Beach, FL, USA.

vanMarle, K., \& Scholl, B. J. (2003). Attentive Tracking of Objects Versus Substances. Psychological Science, 14(5), 498-504. https://doi.org/10.1111/1467-9280.03451

Wilson, J. A., \& Robinson, J. O. (1986). The impossibly twisted Pulfrich pendulum. Perception, 15(4), 503 - 504. https://doi.org/10.1068/p150503

Yi, D.-J., Turk-Browne, N. B., Flombaum, J. I., Kim, M.-S., Scholl, B. J., \& Chun, M. M. (2008). Spatiotemporal object continuity in human ventral visual cortex. Proceedings of the National Academy of Sciences, 105(26), 8840-8845. https://doi.org/10.1073/pnas.0802525105

Xu, F., \& Carey, S. (1996). Infants' Metaphysics: The Case of Numerical Identity. Cognitive Psychology, 30(2), 111-153. https://doi.org/10.1006/cogp.1996.0005 